\title{
A Simple Phase Shifting Technique for an Injection Locked Oscillator
}

\author{
Mohammed Abdul-Niby \\ School of Engineering \\ The Australian College of Kuwait, \\ Kuwait \\ m.nibi@ack.edu.kw
}

\author{
Mamoon Alameen \\ School of Engineering \\ The Australian College of Kuwait, \\ Kuwait \\ m.radiy@ack.edu.kw
}

\author{
Hania Baitie \\ School of Engineering \\ The Australian College of Kuwait, \\ Kuwait \\ h.baitie@ack.edu.kw
}

\begin{abstract}
In Self Oscillating systems, locking of the oscillators can take place for injected signals close in frequency to $\mathbf{n}^{\text {th }}$ harmonics of the free-running frequency. In this paper, we present a simple design for digital phase shift control by using a harmonically injection locked oscillator (ILO) of $35 \mathrm{MHz}$ frequency. Phase shifters at high frequencies are essential in many communication system applications such as frequency synthesis, quadrature signal generation and phase locked loops (PLLs).
\end{abstract} shifter

Keywords-injection locked oscillator; injection locking; phase

\section{INTRODUCTION}

In Self Oscillating systems, oscillators locking can occur when signals close in frequency to nth harmonics of the freerunning frequency are injected [1-5]. The superharmonic injection locked oscillator is designed and implemented using a Colpitts oscillator which has a free-running frequency of $35 \mathrm{MHz}$. This frequency is used as the intermediate frequency (IF) in low capacity FM-FDM and PSK-PCM communication systems. For very practical reasons, the Colpitts oscillator is injected with the 2 nd harmonic signal's frequency i.e. $70 \mathrm{MHz}$ to achieve locking. The frequency range in which injection locking happens is called "locking range". Injection locking also happens when the injected frequency is close to the harmonic or subharmonic of the free-running oscillator frequency i.e. $n \omega_{o}$ or $\omega_{0} / n$ [1-3]. The use of q superharmonic injection-locked oscillator as frequency divider was discussed in [6], whereas a precise digital phase shifter using a phaselocked loop was investigated in [7]. In [8], authors presented a multiphase injection-locking (IL) technique and its application in the locking range extension in multiphase injection-locking oscillators (ILOs) for terahertz (THz) signal generation.

\section{PROPOSED ARCHITECTURE}

The proposed phase shifting technique is shown in Figure 1. By injecting a signal harmonically related to the oscillator free-running frequency, a dual function of locking and phase shifting may be achieved. The magnitude of frequency shift and rate of change of phase is controlled by the pulse amplitude, while the pulse duration controls the length of time for which the total phase change can be obtained.

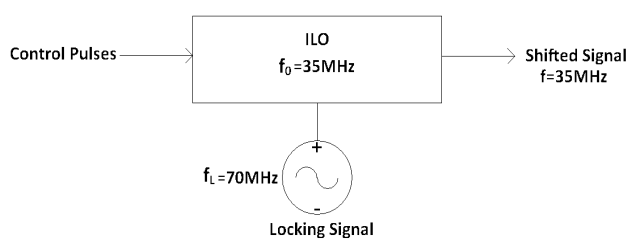

Fig. 1. Injection locked oscillator (ILO) with phase control pulses model

\section{ANALYSIS OF THE PROPOSED PHASE SHIFTING TECHNIQUE}

Figure 2 shows the time domain waveform of the ILO in locked and unlocked conditions. In this method the oscillator is forced to operate outside the locking range. This way of detuning is developed through the use of a varactor diode connected in parallel with the oscillator tuned circuit. The varactor diode act as a variable capacitor when the bias voltage is varied hence controls the oscillator free-running frequency.

The oscillator free-running frequency is controlled by applying a control pulse to the varacor diode. The amplitude of the pulse should cause sufficient detuning to force the oscillator to operate at new frequency outside the locking range. As this condition is achieved, then proper pulse duration should be chosen to obtain the required phase shift such that the condition $T \Delta f=$ constant is valid, where $T$ is the pulse duration an $\Delta f$ is the frequency detuning.

After applying the unipolar pulse to the varactor diode, the oscillator will be running at a phase of $\Delta \varphi$ relative to its previous locked phase. In order to prove that the unipolar pulse can cause the required phase shift, let us find the relation between $T$ and $\Delta f$ that is satisfying our requirement to obtain a phase shift in the ILO frequency.

With reference to Figure 2, we can proceed as follows: 


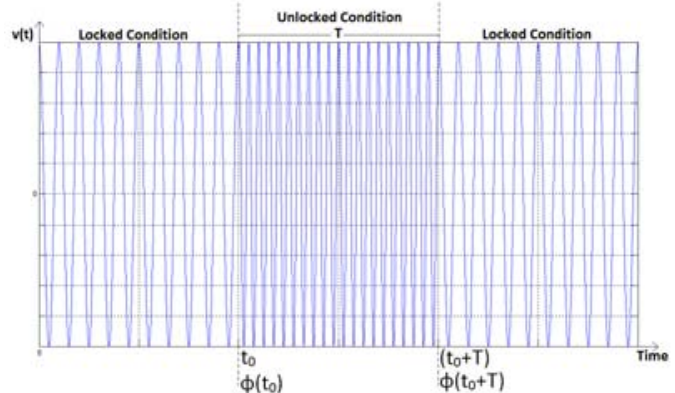

Fig. 2. The time domain waveform of the ILO in locked and unlocked conditions.

For $t<t_{o}$ i.e. before applying the unipolar pulse, the oscillator is in a locked condition and the oscillator locked signal is:

$$
v(t)=V \cos \omega t
$$

And the locking signal is:

$$
v_{L}(t)=V_{L} \cos n \omega t
$$

At the beginning of the pulse at $t=t_{o}$, the ILO is forced to operate at some $(\omega+\Delta \omega)$ frequency outside the locking i.e.

$$
\Delta \omega>\Delta \omega_{\text {Lmax }_{x}} .
$$

The ILO output $v(t)$ becomes:

$$
v(t)=V \cos (\omega+\Delta \omega) t
$$

The phase at the instant when $t=t_{o}^{+}$is:

$$
\varphi\left(t_{o}\right)=(\omega+\Delta \omega) t_{o}
$$

The end of the pulse i.e. at $t=t_{o}+T$, the phase is:

$$
\varphi\left(t_{o}+T\right)=(\omega+\Delta \omega)\left(t_{o}+T\right)
$$

The phase accumulation during the pulse duration $T$ can be found from the difference between equations (4) and (5):

$$
\Delta \varphi=(\varphi)\left(t_{o}+T\right)-\varphi\left(t_{o}\right)=(\omega+\Delta \omega) T
$$

If the pulse had not been applied, the phase accumulation of the ILO output signal for the same time $T$ elapsed would be:

$$
\Delta \varphi_{o}=\omega T
$$

Thus, the resultant phase shift due to $\Delta \omega$ in $T$ can be obtained from equations (6) and (7):

$$
\Delta \Psi=\Delta \varphi-\Delta \varphi_{0}=\Delta \omega T=2 \pi \Delta f T
$$

Clearly, one can see from equation (8) that the phase shift depends on the product of frequency detuning and the pulse duration. For constant phase shift, the named product should be kept constant. Under the continuous drive of the locking signal, the oscillator will return to its locked condition at the end of the pulse and running at frequency $f$ with the new phase.

For immediate (no transient) return to the locked condition, $\Delta \Psi$ must be equal to the integer multiple of the period of the locking signal. Since the later is $2 \pi / \mathrm{n}$ with respect to the ILO signal period $(2 \pi)$, then the following condition must be satisfied:

$$
\begin{aligned}
\Delta \Psi & =k \frac{2 \pi}{n} \\
k & =1,2,3, \ldots .
\end{aligned}
$$

Comparing equation (8) and (9) we find:

$$
\Delta f T=\frac{k}{n}=\text { constant }
$$

\section{CIRCUIT DESIGN AND OPERATION}

Figure 3 shows the schematic diagram of the designed injection locked oscillator. The oscillator is designed to operate at $35 \mathrm{MHz}$; the total capacitance in the oscillator tuned circuit is chosen to be 60PF while the inductance is calculated from the formula of the free running frequency $\omega=1 / \sqrt{L C}$. It is found that $\mathrm{L}=0.35 \mu \mathrm{H}$, thus the coil of 10 turns wounded side by side on a plastic bobbin of $4 \mathrm{~mm}$ diameter with tuning ferrite core. For the ILO oscillator, an integrated circuit UL1111N (14 pins DIL) was used, in such integrated circuit there are five transistors, two of these are in differential pair and three separate transistors. The phase shift control pulses are applied to the varactor (BA124) which is reversed biased by $-6 \mathrm{~V}$ supply through a potentiometer as shown in Figure 3.

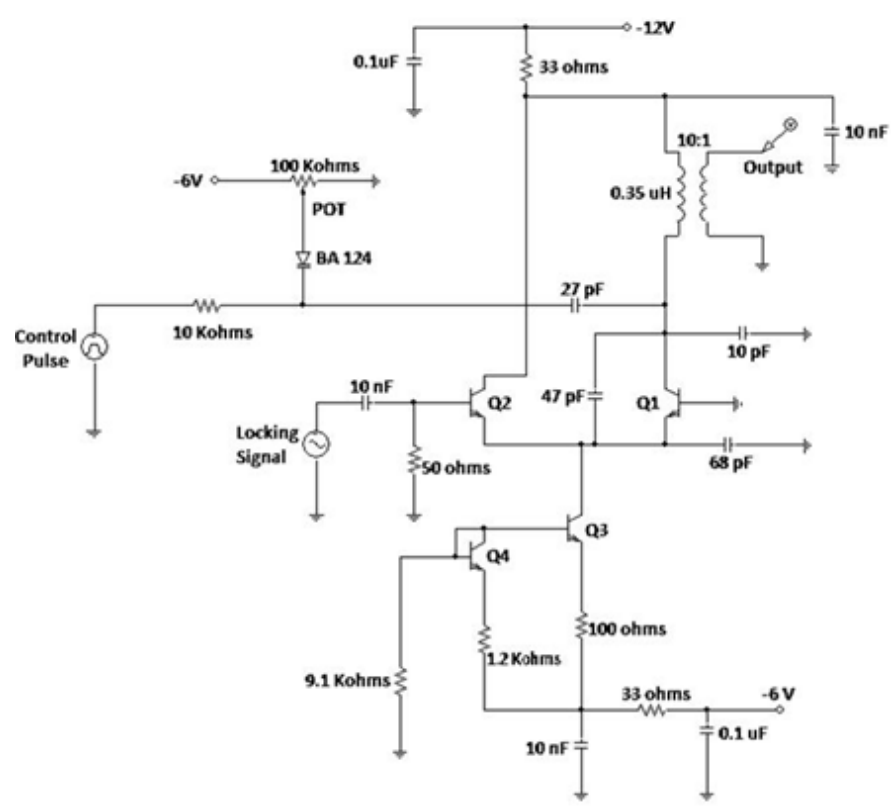

Fig. 3. Schematic diagram of the designed injection locked oscillator with phase control pulses.

\section{EXPERIMENTAL MEASUREMENTS}

It is necessary to conduct a set of static measurements on the proposed ILO circuit to examine its parameters and ability of achieving phase shifting. These measurements are 
essential in setting the proper operation of the phase shift control.

\section{A. Varactor static capacitance-voltage characteristic (BA124)}

The static capacitance-voltage characteristic of the varactor is shown in Figure 4.

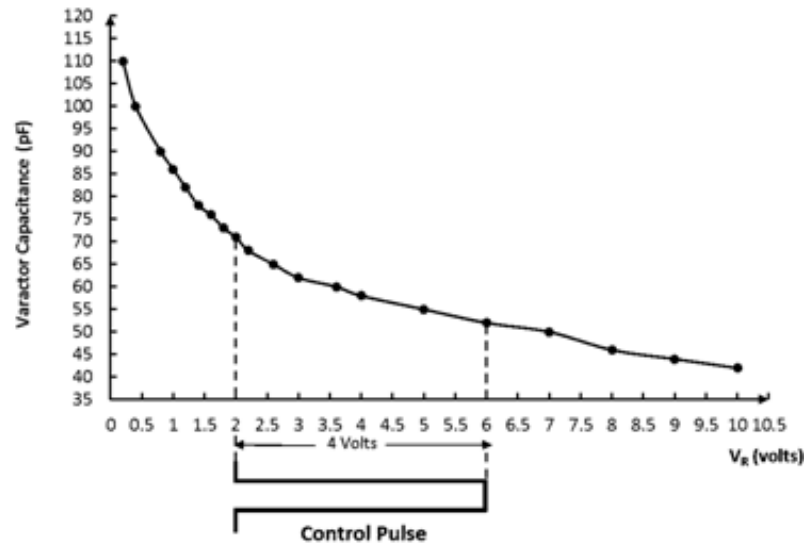

Fig. 4. Static characteristic $\left(\mathrm{C}_{\mathrm{V}}\right.$ vs $\left.\mathrm{V}_{\mathrm{R}}\right)$ of the BA124 Varactor diode. Pulses shown are applied to control the capacitance.

\section{B. Measurement of the ILO locking band as a function of the locking signal voltage}

Measurement of locking band or locking ranges as a function of locking signal voltage is performed for harmonic locking $(n=2)$. Locking band $B_{L}\left(B_{L}=2 \Delta f_{L \max }\right)$ measurements help in deciding what locking signal level should be injected to obtain a locking range less than the fixed frequency detuning caused by the applied control pulse of $4 \mathrm{~V}$ amplitude. The measurement is done by setting zero locking signal voltage and adjusting the varactor operating point at $2 \mathrm{~V}$ by the preset POT (Figure 3). The oscillator free-running frequency is then tuned to $35 \mathrm{MHz}$ by the inductance core, and tuning signal to 70 $\mathrm{MHz}$. Setting the locking signal to a certain voltage $\mathrm{V}_{\mathrm{L}}$ (below the oscillator voltage $V_{O}$ ) starting from a very small locking signal level, then detuning the locking signal and observe the spectrum of the ILO output then recording lower and upper frequencies at which the unlock occur. The frequency limits of locking and locking band curves are plotted in Figures 5 and 6 .

\section{CONCLUSION}

A simple ILO based phase shifting technique is presented. The presented architecture consists of a Colpitts oscillator running at an intermediate frequency of $35 \mathrm{MHz}$ and a $2^{\text {nd }}$ harmonic frequency injected signal. The proposed ILO attains a large locking range of about $0.88 \mathrm{MHz}$.

The proposed design was demonstrated and tested successfully; the obtained results are very satisfactory.
1. The use of unipolar pulses which are preferable due to the possibility of using TTL in processing such pulses.

2. The use of a varactor diode as a controlled reactive element in the oscillator tuned circuit to force the ILO to operate at a frequency outside the looking range.

3. The proposed circuit of the injection locked oscillator can be used frequency divider.

Apart of the above mentioned features, the proposed technique has a disadvantage which is the need of supplying the locking signal at the second harmonic of the oscillator output frequency. This may be impractical especially at high microwave frequencies.

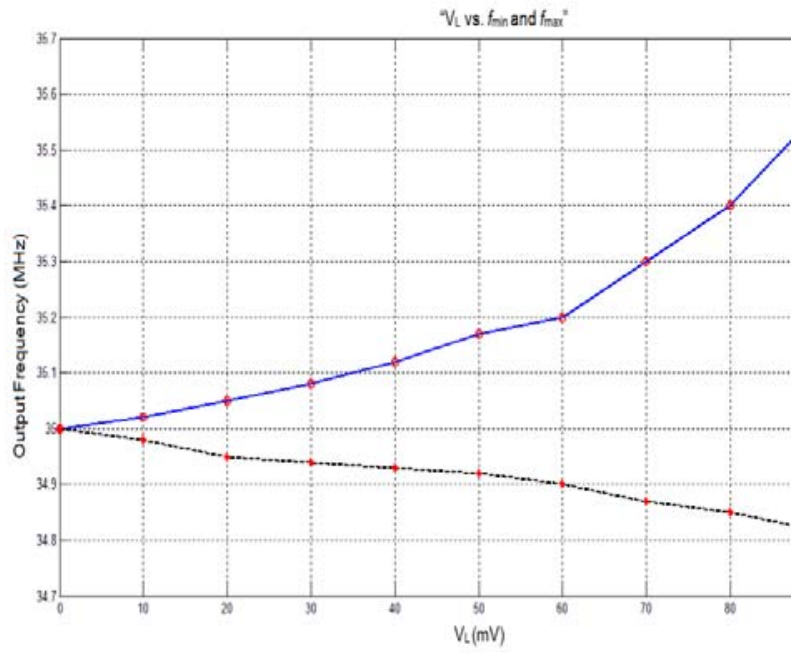

Fig. 5. ILO output frequency versus locking signal voltage.

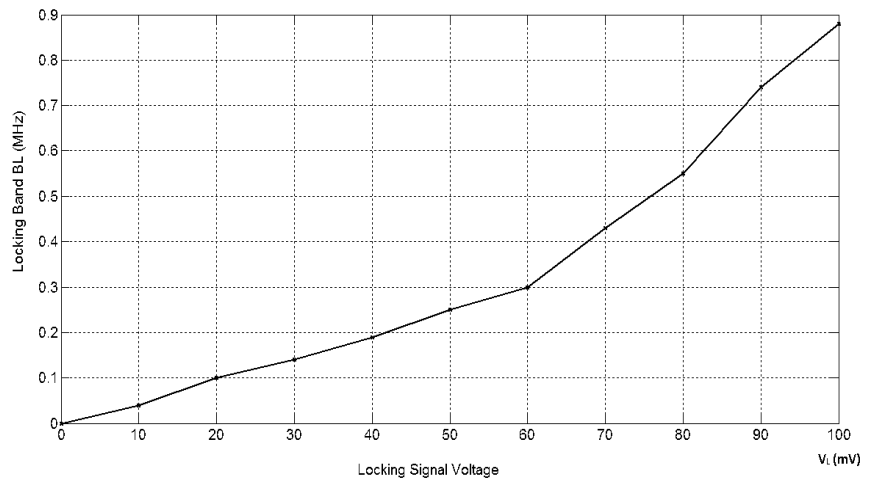

Fig. 6. Locking band as a function of the voltage of the locking signal.

\section{ACKNOWLEDGMENT}

The authors appreciate the continuous support of the Australian College of Kuwait in conducting this research work.

The essential features of this technique are: 


\section{REFERENCES}

[1] R. Adler, "A Study of Locking Phenomena in Oscillators", Proceedings of the IRE, Vol. 34, No. 6, pp. 351-357, 1946

[2] K. Kurokawa, "Injection locking of microwave solid-state oscillators", Proceedings of the IEEE, Vol. 61, No. 10, pp. 1336-1410, 1973

[3] L. J. Paciorek, "Injection locking of oscillators", Proceedings of the IEEE, Vol. 53, No. 11, pp. 1723-1727, 1965

[4] H. L. Stover, "Theoretical explanation of the output spectra of unlocked driven oscillators", Proceedings of the IEEE, Vol. 54, No. 2, pp. 310311, 1966

[5] M. Armand, "On the output spectrum of unlocked driven oscillators", Proceedings of the IEEE, Vol. 57, No. 5, pp. 798-799, 1969
[6] H. R. Rategh, T. H. Lee, "Superharmonic injection-locked frequency dividers", IEEE Journal of Solid-State Circuits, Vol. 34, No. 6, pp. 813821,1999

[7] J. Austin, J. R. Forrest, "A precise digital phase shifter using a phaselocked loop", Electronics Letters, Vol. 14, No. 8, p. 254-255, 1978

[8] H. Wang, K. Sengupta, RF and mm-Wave Power Generation in Silicon, Academic Press, Elsevier, 2016 